\title{
Statistical Properties of Relaxing Dusty Plasmas
}

\author{
CHUTOV Yuriy* and SCHRAM Pieter ${ }^{1}$ \\ Faculty of Radio Physics, Taras Shevchenko Kiev University, \\ Volodymyrs'ka Str. 64, 252017 Kiev, Ukraine \\ 'Department of Physics, Eindhoven University of Technology, The Netherlands
}

(Received: 18 January 2000 / Accepted: 9 June 2000)

\begin{abstract}
The Liouville equation is considered for relaxing dusty plasmas consisting initially of equilibrium electrons and ions as well as neutral dust particles. The plasmas relax then due to a collection of electrons and ions by dust particles. The free and collected electrons and ions are considered as a common subsystem described by the common whole distribution function. It is shown that this sub-system is described by a complex integral-differential equation with Non-Markovian kinetics. The general properties of dusty plasmas are important for controlled fusion devices because dust particles can be created in fusion devices due to the plasma-wall interaction and therefore can influence particle and energy fluxes to the wall.
\end{abstract}

\section{Keywords:}

dusty plasma, Liouville equation, Non-Markovian kinetics, fusion device,

\section{Introduction}

It is established recently [1] that dust particles can be created due to the plasma-wall interaction in fusion devices (divertors, limiters, etc) and can influence particle and energy fluxes to the wall. Therefore it is important for controlled fusion to study plasmas with dust particles (dusty plasmas) including their general properties.

Dusty plasmas are complex statistical systems due to the intensive charge exchange between dust particles and background electrons and ions. Usually these plasmas are considered as statistical systems consisting of at least three sub-systems (components), namely: electrons, ions, and dust particles.

However this consideration is not always sufficient. Indeed, the most important parameter of dust particles is their electric charge, which appears due to a collection of free electrons and ions by dust particles. The bounded electrons and ions creating the dust particle charge are not included in the sub-systems of free electrons and

*Corresponding author's e-mail: yuch@boy.rpd.univ.kiev.ua ions after their collection by dust particles although they act on free particles in exactly the same way as before collection. This circumstance renders the consideration mentioned above somewhat unlogical, especially in the case of non-stationary dusty plasmas.

In the framework of the consideration of dusty plasmas as statistical systems with separated subsystems, non-stationary dusty plasmas have to be considered as statistical systems with variable numbers of particles [2] due to the collection of background electrons and ions by dust particles. In the general case, such non-stationary systems have to be non-equilibrium because the probabilities of all processes leading to a change of particle numbers depend on particle energies. In particular, a selective collection of electrons and ions by dust particles depending on electron and ion energy can cause a non-equilibrium state of dusty plasma [3]. However, modern theoretical investigations of dusty plasmas including their non-stationary behaviour [4-6]

C2000 by The Japan Society of Plasma Science and Nuclear Fusion Research 
are usually carried out on basis of the assumption of equilibrium background electrons and ions, because of the large complications of a non-equilibrium description.

The aim of this work is a consideration of relaxing dusty plasmas as integral statistical systems. Some results of these investigations were published earlier in $[7,8]$.

\section{Statistical description}

Let us consider a system of $N_{e}$ electrons and $N_{i}$ ions (or $N=N_{e}+N_{i}$ particles) which is described by a whole distribution function $f_{N}(X, t)$ where $X$ is the whole set of

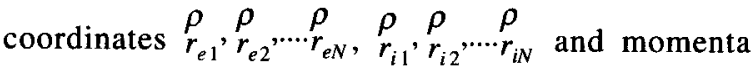

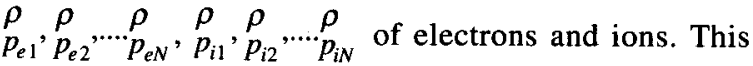
system is relaxing due to a collection of electrons and ions by $M$ immobile identical grains (dust particles) in a volume containing these electrons and ions. In this case electrons and ions can be divided into two groups which change in time, namely: $K_{e}(t)$ and $K_{i}(t)$ free electrons and ions respectively as well as $L_{e}(t)$ and $K_{i}(t)$, i.e. electrons and ions coupled to grains. As a result, the following relations hold:

$$
K_{\alpha}(t)+L_{\alpha}(t)=N_{\alpha} .
$$

Index $\alpha=e, i$ denotes electrons and ions.

According to this division, the total potential energy $E_{\alpha, \beta}=\frac{1}{2} \sum_{\substack{1 \leq \alpha j, \beta_{k} \leq N_{\alpha} \\ j \neq k}}\left(\phi / \stackrel{\rho}{r}_{\alpha}-{\underset{r}{\beta_{k}}}_{\rho}^{\rho}\right)$ of electronelectron, electron-ion and ion-ion interaction can be divided into several kinds, namely: the potential energy of interactions between free particles $\left(E_{o f, \beta_{f}}\right)$, between free and bounded particles $\left(E_{o f, \beta b}\right)$, and between bounded particles $\left(E_{\alpha b, \beta b}\right)$. Indexes $\alpha, \beta=e, i$ denote here electrons and ions, indexes $f$ and $b$ denote free and bounded particles, respectively.

As a result, the Hamiltonian can be written in the following form:

$$
\begin{aligned}
& \left.H_{N}(X)=\sum_{1 \leq \alpha_{j j} \leq N_{\alpha}}\left(\begin{array}{l}
\rho_{2} \\
\frac{P_{\alpha j}}{2 m}+U_{o}\left({ }_{r_{\alpha j}}^{\rho}\right.
\end{array}\right)\right) \\
& +\sum_{1 \leq \alpha j b \leq N_{\alpha}}\left(\frac{\rho_{2}}{P_{\alpha j}} \frac{U_{o}}{2 m}\left({\stackrel{\rho}{r_{\alpha j}}}\right)\right)+E_{\alpha f, \beta f} \\
& +E_{\alpha f, \beta b}+E_{\alpha b, k b} \text {, }
\end{aligned}
$$

where these energies can be written as

$$
\begin{aligned}
& E_{\alpha f, \beta f}=\frac{1}{2} \sum_{\substack{1 \leq \alpha j f, \beta k f \leq N_{\alpha} \\
j \neq k}}\left(\phi /{\stackrel{\rho}{r_{\alpha j}}}_{\substack{\rho \\
r_{\beta k}}}^{\rho}{\stackrel{\rho}{r_{\beta k}}}^{\prime}\right), \\
& E_{\alpha f, \beta b}=\frac{1}{2} \sum_{\substack{1 \leq \alpha_{j f}, \beta k b \leq N_{\alpha} \\
j \neq k}}\left(\phi /{\stackrel{\rho}{r_{\alpha j}}}_{\alpha_{j}}^{\rho}-{\stackrel{\rho}{r_{k}}}^{\prime}\right), \\
& E_{\alpha b, \beta b}=\frac{1}{2} \sum_{\substack{1 \leq \alpha j b, \beta k b \leq N_{\alpha} \\
j \neq k}}\left(\phi /{\stackrel{\rho}{r_{\alpha j}}}_{\alpha_{j}}^{\rho}-{\stackrel{\rho}{r_{\beta k}}}^{\rho}\right)
\end{aligned}
$$

Triple indexes are necessary here for distinguishing free (index $f$ ) and bounded (index $b$ ) electrons and ions at the condition of the conservation of double indexes $(\alpha j, \beta k)$ for the equations of free particle motion.

Let us introduce the number $Q_{o m}\left(R_{o m}, t\right)$ of electrons and ions collected to some grain to the instant $t$ so that the effective charge $Q_{\alpha m}\left(R_{\alpha m}, t\right)$ of a grain can be written as:

$$
Q_{m}\left(R_{m}, t\right)=\sum_{\alpha} Q_{\alpha_{m}}\left(R_{\alpha_{m}}, t\right)
$$

Obviously, the following relation is valid:

$$
L_{\alpha}(t)=\sum_{1 \leq m \leq M} Q_{\alpha_{m}}\left(R_{m}, t\right)
$$

Let us also introduce the effective inner energy $E_{m}\left(R_{m}, t\right)$ of a grain including the kinetic energy of bounded particles as well as the potential energy of the interaction between particles bounded by the grain, and the potential energy of these bounded particles in external fields. Then we have:

$$
\begin{aligned}
& \sum_{1 \leq m \leq M} E_{m}\left(R_{m}, t\right)=\sum_{1 \leq \alpha j b \leq N_{\alpha}}\left(\begin{array}{l}
\rho_{2} \\
\frac{P_{\alpha j}}{2 m}+U_{o}\left({ }_{r_{\alpha j}}^{\rho}\right)
\end{array}\right) \\
& +\frac{1}{2} \sum_{\substack{1 \leq \alpha j b m, \beta k b m \leq N_{\alpha} \\
j \neq k}} \phi\left(/{ }_{r_{\alpha j}}^{\rho}-{\stackrel{\rho}{r_{\beta j}}}^{\rho}\right)
\end{aligned}
$$

Here indexes $\alpha \mathrm{jbm}$ and $\beta \mathrm{jbm}$ denote the electrons and ions bounded by the grain $m$.

Then the equation (2) can be rewritten in the following form: 


$$
\begin{aligned}
& H_{N}(X)=\sum_{1 \leq \alpha j f \leq N_{\alpha}}\left(\begin{array}{l}
\rho_{2} \\
\frac{P_{\alpha j}}{2 m}+U_{o}\left({ }_{r_{j j}}\right)
\end{array}\right) \\
& +\frac{1}{2} \sum_{\substack{1 \leq \alpha j b m, \beta k b m \leq N_{\alpha} \\
j \neq k}} \phi\left(/ \rho_{\alpha_{j}}-\rho_{r_{\beta k}}\right)+\sum_{1 \leq m \leq M} E_{m}\left(\rho_{R_{m}}^{\rho}, t\right) \\
& +\frac{1}{2} \sum_{\substack{1 \leq i f \leq N_{\alpha} \\
1 \leq m \leq M}} Q_{m}\left({\left.\stackrel{\rho}{R_{m}}, t\right) \phi\left(/ \rho_{i f}-\rho_{R_{m}}\right)}^{\rho}\right) \\
& +\frac{1}{2} \sum_{\substack{1 \leq m, n \leq M \\
m \neq n}} Q_{m}\left(\stackrel{\rho}{R_{m}}, t\right) Q_{n}\left(\stackrel{R}{R}_{n}^{\rho}, t\right) \phi\left({ }_{R_{m}}^{\rho}-\stackrel{\rho}{R}_{n}^{\prime}\right) .
\end{aligned}
$$

It is very important that this Hamiltonian is constant in time, although free and bounded particles have variable potential and kinetic energies. This constancy is provided by the exchange between free and bounded particles. This means that the effective charge and inner energy of dust particles depend nondirectly (through time) on the set of coordinates

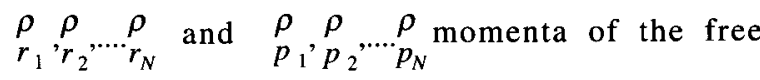
particles.

Taking into account equation (15), it is possible to express the Liouvile equation

$$
\frac{\partial f_{N}}{\partial t}+\sum_{1 \leq i \leq M}\left(\frac{\partial H}{\partial P_{i}} \frac{\partial f_{N}}{\partial r_{i}}-\frac{\partial H}{\partial r_{i}^{\rho}} \frac{\partial f_{N}}{\partial P_{i}}\right)=0
$$

as the following formal equation for the whole distribution function $f_{N}(X, t)$ :

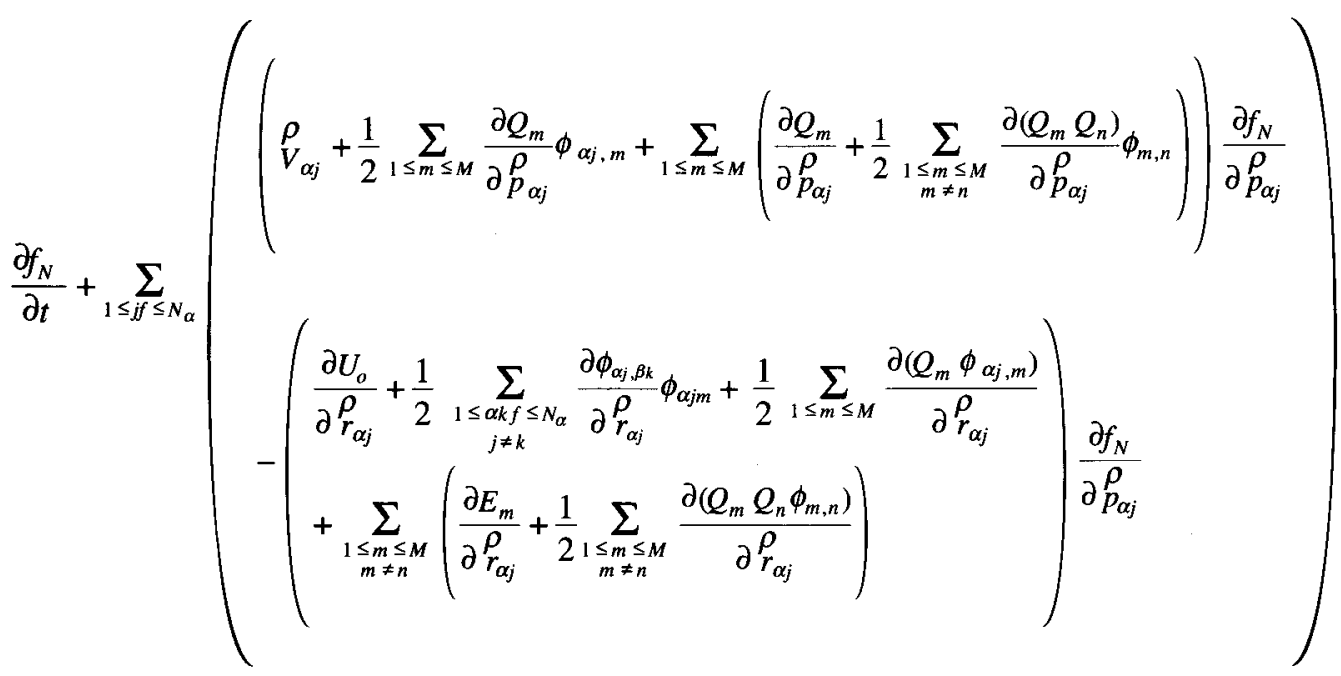

It should be noted that the three last terms in equation (7) depend on the whole distribution function $f_{N}(X, t)$ and therefore on the whole set of coordinates

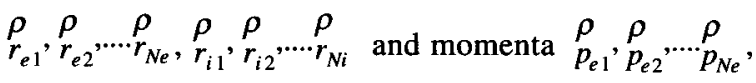
${ }_{p_{i 1}}^{\rho}, p_{i 2}, \cdots . . p_{N i}^{\rho}$ as well as on the positions $R_{m}$ of a grain. These integral dependencies are very complex, as can be seen, for example, from the following expression for the number of particles $Q_{m}\left(R_{m}, t\right)$ collected by some grain during the time $t$ :

$$
\begin{aligned}
& Q_{m}\left(R_{m}, t\right) \\
& =\sum_{1 \leq \alpha_{j} \leq K_{\alpha}(t)} \int_{X^{*}, t} w\left(X_{\alpha_{j}}, R_{m}\right) f_{N}(X, t) d X d t,
\end{aligned}
$$

where $w\left(X_{\alpha j}, R_{m}\right)$ is the probability that a free $\alpha j$-particle is collected by a grain located at point $R m$. Therefore equation (19) is a complex integral-differential equation together with an equation (20) can be expected to lead to non-Markovian kinetics.

The integration of equation (10) leads in principle to the possibility to derive equations for reduced distribution functions including the equation for the oneparticle distribution function, such as the Boltzman equation with a collision integral. However this integration is very complex in general and therefore it is very useful for the investigation of relaxing plasmas to use computer simulation allowing to trace the evolution of every computer particle during the relaxation of dusty plasmas. 


\section{Conclusion}

The theoretical treatment of dusty plasmas relaxing due to a collection of electrons and ions by dust particles shows that the free and collected electrons and ions can be considered as a common sub-system described by the common whole distribution function. It is shown that this sub-system is described by a complex integral-differential equation with Non-Markovian kinetics.

\section{Acnowledgments}

This work was partially supported by INTAS (Contract No 96-0617) and by a grant from the Ukrainian Committee of Science and Technology.

\section{References}

[1] J. Winter, Plasma Phys. Cont. Fusion. 40, 1201 (1998).

[2] R. Bowley and M Sancher, Introductory statistical mechanics, (Oxford: Clarendy press, 1996).

[3] Yu. Chutov et al., Physica B 228, 11 (1996).

[4] H.C. Lee et al., Phys. Rev. E56, 4596 (1997).

[5] Tsytovich V.N., Uspekhi fisicheskikh nauk 167, 57 (1997). (in Russian)

[6] Wang, X. et al.,Phys. Plasmas 4, 3759 (1997).

[7] Chutov Yu et al., In: Strongly Coupled Coulomb Systems: (Plenum Press, 1998).

[8] Chutov Yu et al., Contrib. Plasma Phys. 39, 127 (1999). 